\title{
Application of the Measurement System Based on Phase Shifting Using Digital Fringe in Locomotive Casting Mold Detection
}

\author{
Xiaoming Zhou ${ }^{1,2}$ \\ ${ }^{1}$ School of Materials Science and Engineering, Central South University \\ Changsha, China \\ ${ }^{2}$ Zhuzhou Lince Group Co.,Ltd \\ Zhuzhou, China \\ flying803@126.com
}

\begin{abstract}
The acquirement of 3D geometric information is the most important for reverse engineering. With these advantages such as no phase shift error, rapid measurement, easy to carry and high precision, the three-dimensional(3D) measurement system which is based on phase shifting technology using digital fringe based DLP projection has been widely used in the object three-dimensional shape measurement.The precision of camera calibration determines the measurement accuracy of the system. In this paper, an improved flexible camera calibration means is presented than Zhang's planar flexible calibration method. By applying the phase-shift technique to fringe projection, point clouds with high spatial resolution and limited accuracy can be generated.In the locomotive casting mold design and processing, error detection of the mold is a very important step. In this paper the principle of the measurement system based phase shifting using digital fringe and the algorithm of ICP is discussed, which is used in the locomotive casting mold design and error detection.
\end{abstract}

Keywords-locomotive casting mold; phase shifting; digital fringe; camera calibration; ICP algorithm

\section{INTRODUCTION}

Reverse Engineering generally refers to through a variety of measurements, in view of the existing product samples, utilizing 3D digital measuring instruments measured accurately and rapidly the contour coordinates and reconstituting the three dimensional CAD surface model, transform The original material object into threedimensional digital model of computer, and optimize the model analysis and processing, also can redesign on this basis [1]. The acquirement of 3D geometric information acquisition is very important work for reverse engineering. Reverse engineering scanning technology, based on phase shifting fringe measuring principle, has faster speed and higher precision than laser scanning[2]. Therefore, it can greatly shorten the product development cycle and improve the quality of the products in practical application of industrial production, especially can greatly shorten the development cycle of mold in the locomotive casting mold design, testing and manufacturing process.

\section{BASED ON PHASE SHIFTING MEASUREMENT PRINCIPLE OF REVERSE ENGINEERING SCANNING TECHNOLOGY}

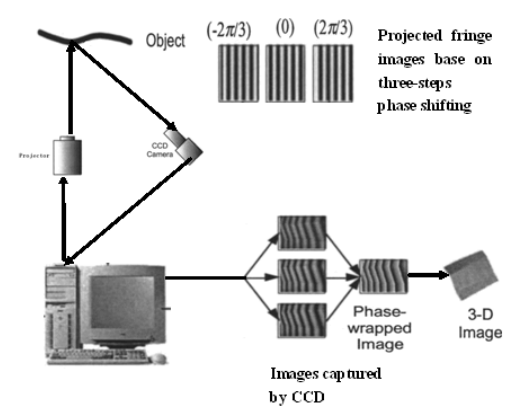

Figure 1. Measurement principle diagram

\section{A. Camera calibration Algorithm}

The precision of three-dimensional measuring system is determined by Camera calibration accuracy [3-4]. Until now, many calibration methods have proposed by the domestic and foreign scholars. Brown [5] put forward a fully nonlinear calibration model of camera, which required three-dimensional calibration object in the calibration process. As a result of the full non-linear model, the solution is instable. What is more, the calibration needs a 3D calibration object with high accuracy, which is usually more expensive. A classic two-step calibration means, which makes the solving process, be simple and stable is proposed by Tsai [6]. However, only the firstorder radial distortion is considered, and Tsai's camera calibration also required 3D calibration object. Based on the method that Triggs and Zisserman proposed, Zhang Zhengyou [7] came up with a method that only needed a planar grid calibration target, but the first-order radial distortion is only considered in Zhang's method and the model is relatively rough. It has certain drawbacks to improve the accuracy for the calibration targets used in these ways above. So in this paper An improved flexible camera calibration algorithm is brought up, and which has been applied to the digital fringe three-dimensional measurement system.

First of all, applying the image coordinates and the corresponding three-dimensional coordinate of the middle 
35 centers in each calibration image, the initial value of external and internal parameters without distortion can be obtained by the following formula.

$$
M I N=\sum_{i=1}^{n} \sum_{j=1}^{m}\left\|m_{i j}-\hat{m}\left(A, R_{i}, t_{i}, M_{j}\right)\right\|^{2}
$$

Where $i$ represent the number of images, $j$ is the number $j$ point in image $i, \hat{m}\left(A, R_{i}, t_{i}, M_{j}\right)$ mean the projection points of image $i$ corresponding to space plane 3D points $M_{j}$. Generally, using the Levenberg-Marquardt algorithm to conduct optimization can get the final external and internal parameters.

Analogously, On account of the distortion in the middle part of images is very small, the initial internal and external camera parameters, distortion parameters can also be got by applying Levenberg-Marquardt algorithm. The optimization algorithm formula as follows:

$$
M I N=\sum_{i=1}^{n} \sum_{j=1}^{m}\left\|m_{i j}-\hat{m}\left(\mathrm{k}_{1}, \mathrm{k}_{2 i}, \mathrm{p}_{1}, \mathrm{p}_{2}, \mathrm{~A}, \mathrm{R}_{i}, \mathrm{t}_{i}, M_{j}\right)\right\|^{2}
$$

Where $k_{1}, \mathrm{k}_{2}, \mathrm{p}_{1}$ and $\mathrm{p}_{2}$ are respective radial and tangential distortion coefficients. Using the result optimized by (2) as a starter, and then plugging all center coordinates of each calibration image into equation (2) to refine parameters, finally, the final internal and external camera parameters and distortion parameters can be calculated.

In order to confirm whether the proposed algorithm improves the accuracy of camera calibration, camera calibration is accomplished using 13 plane target images in this paper. Camera Calibration results are shown in Table 1. The distribution of projection errors is shown in the Fig . 2. The standard deviation of calibration results is [0.02478, 0.02166]. However, Zhang's standard deviation of calibration results is $[0.11688,0.11490]$, which was only considered the first-order radial distortion and used calibration board with checkerboard pattern. The camera calibration method is better than Zhang's method accuracy and stability.

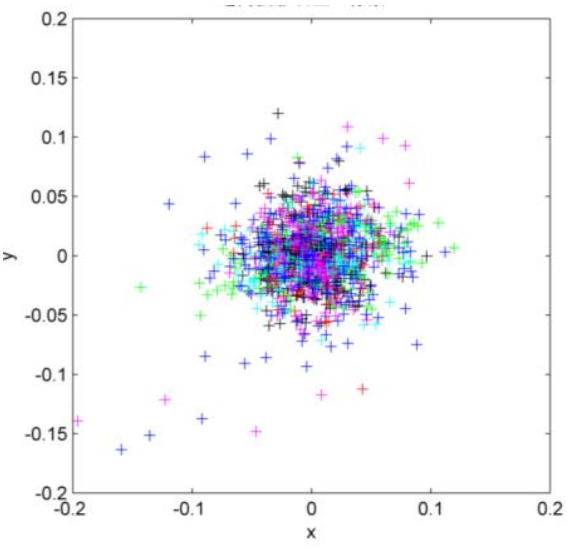

Figure 2. Projection errors distribution

\section{B. Phase unwrapping}

The principle of fringe phase shifting measurement is using white light as light source projecting fringe pattern onto a surface object to modulate. Using relevant demodulation method to carry the object depth information of phase demodulation modulated fringe Be modulated stripe object depth of information carried by adopting relevant demodulation method will be out of phase demodulation. According to the phase and the depth of the relationship. Get the 3D information objects. A typical measurement system composition principle is shown in Fig.1.

After years of development, Phase shift method directly in the spatial domain for image processing has been mature and widely used in optical measurement technology. Three-step phase shifting algorithm requires minimal stripes picture in the optical measurement Therefore, a great advantage of this algorithm is fast[8]. Three-step phase shifting algorithm acquires three sinusoidal fringe patterns in this project, and phase difference is $2 \pi / 3$. Three light intensity function of sinusoidal fringe pattern are as follows respectively:

$$
I_{1}(x, y)=I^{\prime}(x, y)+I^{\prime \prime}(x, y) \cos [\varphi(x, y)-2 \pi / 3]
$$

$I_{2}(x, y)=I^{\prime}(x, y)+I^{\prime \prime}(x, y) \cos [\varphi(x, y)]$

$$
I_{3}(x, y)=I^{\prime}(x, y)+I^{\prime \prime}(x, y) \cos [\varphi(x, y)+2 \pi / 3](5)
$$

$I^{\prime}(x, y)$ is the average intensity, $I^{\prime \prime}(x, y)$ is intensity modulation, $\varphi(x, y)$ is phase function. By type (3) to (5) to (6):

$$
\varphi(x, y)=\tan ^{-1}\left(\sqrt{3} \frac{I_{1}-I_{3}}{2 I_{2}-I_{1}-I_{3}}\right)
$$

The phase function is between 0 to $2 \pi$, the saw-tooth stripe pattern is the multiple cycles of fringe. Restoring continuous monotonous phase diagram should use solution phase to remove the saw-tooth discontinuity [9]. The phase diagram can be converted into three coordinate values ( $\mathrm{x}, \mathrm{y}$, z) by the means of the system calibration algorithm after the completion of the solution phase[10]. 


\section{BASED ON THE CASTING MOLD DETECTION TECHNOLOGY AND APPLICATION ICP ALGORITHM}

\section{A. Icp algorithm}

ICP algorithm is its original iterative closest point matching algorithm. ICP algorithm is essentially based on the optimal matching method of least squares method, it repeats process about "determine the correspondence between point set to calculate the optimal rigid transformation" until convergence criteria of certain correct match have been satisfied. The problem based on ICP algorithm belongs to multi-view alignment problems, depending on the mathematical definition of multialignment can be described as follows: given 3D data point set of two different coordinate system to find out spatial alternation for the two-point so that they can be suitably space matched. Assumed that $\{\mathrm{Pi} \mid \mathrm{Pi} \in \mathrm{R} 3, \mathrm{i}=1,2, \ldots, \mathrm{N}\}$ denotes the first set of points, $\{\mathrm{Qi} \mid \mathrm{Qi} \in \mathrm{R} 3, \mathrm{i}=1,2, \ldots$, $\mathrm{M}$ \}represent the second set of points, Alignment matching conversion of two point sets is to make the following objective function minimum[11]:

$$
f(R, T)=\sum_{i=1}^{n}\left\|P_{i}^{k}-\left(R P_{i}+T\right)\right\|^{2}=\min
$$

The purpose of the ICP algorithm is to find the rotation $\mathrm{R}$ and translation $\mathrm{T}$ between the model data (igs format data) and real objective data (point cloud data) so that two matching data can satisfy the optimal matching of some measurement criteria. Algorithm mainly divides into two parts: to calculate the closest point pair among data and the rigid transformation between the corresponding points set[12]. Assume that the data model represented by X, the object data represented by $\mathrm{P}, \mathrm{X}$ and $\mathrm{P}$ use threedimensional coordinate data collection point representation, respectively.

First, the object coordinates is expressed as $\left\{\mathrm{P}_{\mathrm{i}} \mid \mathrm{P}_{\mathrm{i}} \in \mathrm{R}^{3}\right.$, $\mathrm{i}=1,2, \ldots, \mathrm{Np}\}$ and model coordinates is expressed as $\left\{\mathrm{Q}_{\mathrm{i}} \mid\right.$ $\left.\mathrm{Q}_{\mathrm{i}} \in \mathrm{R}^{3}, \mathrm{i}=1,2, \ldots, \mathrm{N}_{\mathrm{q}}\right\}$, Calculating the object coordinates corresponding to the the corresponding points coordinates the k-th iteration of $\left\{Q_{i}{ }^{k} \mid Q_{i}{ }^{k} \in R^{3}, i=1,2, \ldots\right.$, $\mathrm{N}_{\mathrm{g}}$, caculating the transformation matrix between $\mathrm{P}$ and $\mathrm{Q}^{\mathrm{k}}$, and updating the original transformation until the average distance among the data is less than a given threshold $\tau$, so equation 7 is minimal.

The steps are:

(1)Take the point set $P_{i}^{k} \in P$ on an object coordinate system;

(2) Calculating the corresponding points of model data $Q_{i}^{k} \in Q^{k}$, making $\left\|Q_{i}^{k}-P_{i}^{k}\right\|=\min$;

(3) Calculating the rotation matrix $R_{k}$ and $T_{k}$, so that $\sum_{i=1}^{n}\left\|R^{k} P_{i}^{k}+T^{k}-Q_{i}^{k}\right\|=\min$; $\in P\}$;

(4) Calculating $P^{k+1}=\left\{P_{i}^{k+1} \mid P_{i}^{k+1}=R^{k} P_{i}^{k}+T^{k}, P_{i}^{k}\right.$

$$
\text { (5) Calculating } d^{k+1}=\left(\sum_{i=1}^{n}\left\|p_{i}^{k+1}-q_{i}^{k}\right\|\right) / n \text {; }
$$

(6) If $d_{k+1}$ is not less than a given threshold value $\tau$, returns (2), until the $d_{k+1}<\tau$;

The third step of the ICP algorithm is to optimize the analytical method used to calculate conversion between $P^{k}$ and $Q^{k}$ and match the two corresponding points in two models. Optimal matching parameters of points set obtained through the iterator. in each iteration, the process of matching the corresponding space points between two model only consider the cases of the rigid body transformation relationship between $P^{k}$ and $Q^{k}$, namely $Q^{k}=R P^{k}+T, \mathrm{R}$ is $3 \times 3$ rotation matrix, $\mathrm{T}$ is $3 \times 1$ translation vector, finally, minimize the objective function equation 7 .

\section{B. Casting mould inspection application based on ICP} algorithm

The template is used to format your paper and style the text. All margins, column widths, line spaces, and text fonts are prescribed; please do not alter them. You may note peculiarities. For example, the head margin in this template measures proportionately more than is customary. This measurement and others are deliberate, using specifications that anticipate your paper as one part of the entire proceedings, and not as an independent document Please do not revise any of the current designations.

\section{CASTING MOULD INSPECTION APPLICATION BASED ON ICP ALGORITHM}

Before you begin to format your paper, first write and save the content as a separate text file. Keep your text and graphic files separate until after the text has been formatted and styled. Do not use hard tabs, and limit use of hard returns to only one return at the end of a paragraph. Do not add any kind of pagination anywhere in the paper. Do not number text heads-the template will do that for you.

Finally, complete content and organizational editing before formatting. Please take note of the following items when proofreading spelling and grammar:

Casting mold testing is the process of error analysis. For example, we can use the reverse scanning device to get the 3D entity data in the objects of casting mould manufacturing process. Entering The scanned data and the original data of casting mold obtained by CAD design into the computer at the same time and the original data is as a baseline for comparison . the relationship between the two kinds of data has shown intuitively. Then, just adjust the corresponding parts in the mold processing appropriately. Not only reduce the production cycle but also reduce the processing cost. As shown in Fig .3, Fig .4, Fig .5, Fig .6.

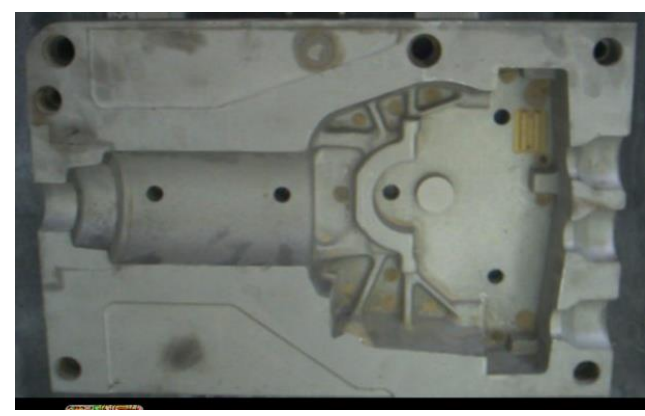

Figure 3 locomotive components for a casting mould entity 


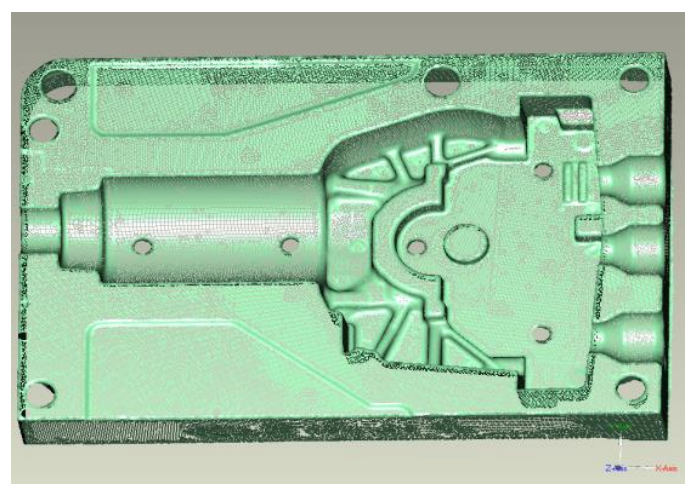

Figure 4 mold reverse scanning point cloud data

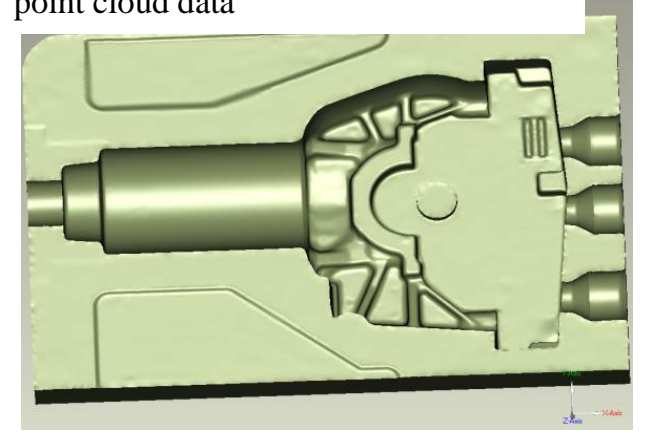

Figure 5 mold CAD

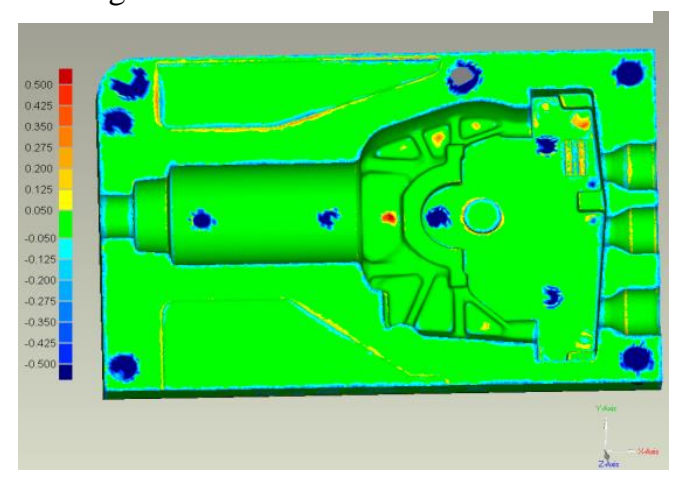

Figure 6 mold color map scanning point clouds and CAD data test result

\section{CONCLUSION}

Reverse engineering, as an important technology, has important uses in the locomotive mold product design, manufacturing and testing areas, also can significantly shorten product development cycles, reduce development risk and cost of the products. This paper discussed that practice test of the locomotive casting molds achieved good results and economic benefits.

\section{REFERENCES}

[1] Xianjie Chen, Xiaobei Li,Min Li, RP \& M Technology Development in China, the National Productivity Centre Network, 2001

[2] Dahui Qin, Congjun Wang, Shuhuai Huang, Based on machine vision 3D laser line scanning system, China Foundry Machinery \& Technology, 2005: 320-21

[3] Qin Dahui, Li Zhongwei, Wang Congjun (2009) "3-D Shape Measurement of Complex Objects by Combining Color-Coded Fringe and Neural Networks," Tsinghua Science and Technology, Vol. 14, pp 66-70.

[4] Zhan Song, Ronald Chung (2008) "Use of LCD Panel for Calibrating Structured-Light-Based Range Sensing System," J IEEE TRANSACTIONS ON INSTRUMENTATION AND MEASUREMENT, pp 1-8.

[5] Brown, D.C. (1971) "Close-range camera calibration," Photogrammetric Engineering and Remote Sensing, Vol. 37, No. 8, pp 855-866.

[6] R. Y. Tsai (1987) "A versatile camera calibration technique for high accuracy 3D machine vision metrology using off-the-shelf TV cameras and lenses," IEEE J. Robot. Autom., Vol. RA-3, No. 4, pp 323-344.

[7] Z. Zhang (2000) "A flexible new technique for camera calibration," IEEE Trans. Pattern Anal. Mach. Intel., Vol. 22, No.11, pp 1330 1334.

[8] D. Malacara, ed., Optical Shop Testing (John Wiley and Songs, NY, 1992).

[9] D.C. Ghiglia and M.D. Pritt, Two-Dimensional Phase Unwrapping: Theory, Algorithms, and Software (John Wiley and Sons, Inc, 1998).

[10] Q. Hu, PS Huang, Q. Fu, and FP Chiang, " Calibration of a 3D surface contouring and ranging system, " Proc. SPIE 3835, 158-166 (1999)

[11] Tao Jin, Shuiguang Tong, Yongnian Yan, Reverse Engineering,,Beijing: Mechanical Industry Press, 2003: 1

[12] Xiaolin Pan, Three-dimensional Surface Matching Technology: [master's thesis], Nanjing, Nanjing University of Aeronautics and Astronautics Library, 2004.3 